\title{
Correction to: Chlorhexidine - a commonly used but often neglected culprit of dialysis associated anaphylactic reactions (case report)
}

Jia Neng Tan ${ }^{*}$, Yi Da, Sabrina Haroon and Titus Lau

\author{
Correction to: BMC Nephrology (2022) 23:18 \\ https://doi.org/10.1186/s12882-021-02646-x \\ Following publication of the original article [1], the \\ authors identified an error in the author name of Yi DA. \\ The incorrect author name is: Yi. \\ The correct author name is: Yi DA. \\ The author group has been updated above and the orig- \\ inal article [1] has been corrected.
}

Published online: 25 January 2022

\author{
Reference \\ 1. Tan, et al. BMC Nephrology. 2022;23:18. https://doi.org/10.1186/ \\ s12882-021-02646-x.
}

